The Egyptian Journal of Hospital Medicine (July 2018) Vol. 73 (2), Page 6028- 6034

\title{
Evaluation of the Effect of Diet Therapy in the Management and Prevention of Acne Vulgaris
}

\author{
Sultan Mousa Bakri ${ }^{1}$, Alshafie Ali Yahya A $^{2}$, Mohammed Abdulkhaleq Farea ${ }^{3}$, Bakr Abobakr \\ Albrakati $^{4}$, Tharaa Waleed Rambo ${ }^{4}$, Fatimah Mustafa AlShakhs ${ }^{5}$, Aldhafeer Eman Saad $A^{5}$, \\ Rawan Adel shafaay ${ }^{6}$, Mahabbat Nadin Anam A ${ }^{7}$, Njoud Ibrahim Al-Nodali ${ }^{8}$, Yara Mahdi BinSaleh ${ }^{9}$
}

Prince Mohammad Bin Naser Hospital, 2- National Guard Hospital_Jeddah, 3- Hadramout University

College of Medicine, 4- Umm Al-Qura University, 5- Imam Abdulrahman Bin Faisal University, 6Almareefa College, 7- King Saud Bin Abdulaziz University For Health Sciences, 8- University of Hail, 9Alfaisal University

\begin{abstract}
Background: Acne is a widespread and complex skin disease among developed nations, affecting nearly all young adults between the ages of 15 to 17 years. It is a chronic inflammatory skin disorder resulting in psychological stress. Historically, the relationship between diet and acne is controversial. As a result, significant studies have been done to evaluate this relationship. Assessment of these literatures will help to provide better outcomes for the patients.

Objective:This study aims to: 1- Measuring effect of diet and nutritional substances in the management and prevention of acne vulgaris. 2- Providing a reference paper analyzing all the clinical studies in this field. 3- Analyzing the last progression has been reached.

Methods: PubMed database was used for articles selection. We included all relevant articles to our review with the following topics (("Acne Vulgaris/diet therapy"[Mesh]) AND ("Acne Vulgaris/prevention and control"[Mesh])) AND ("Acne Vulgaris/therapy"[Mesh]). We excluded other articles which are not related to this field. The data will be extracted according to specific form in which it is going to be reviewed by group members to assess the dietary effect of nutritional substance on acne vulgaris.

Conclusion: We concluded that there are studies that showed promising results among patients and proved that associations do exist. Nevertheless, further studies with bigger sample and better protocols are needed to establish a concrete evidence-based relationship.
\end{abstract}

Key words: Diet Therapy, Management, Prevention, Acne Vulgaris

INTRODUCTION

Acne vulgaris is one of the most common chronic skin diseases worldwide ${ }^{(1)}$, with over $90 \%$ of males and $80 \%$ of females affected by the age of 21 years ${ }^{(2)}$. Acne involves blockage and/or inflammation of pilosebaceous units (hair follicles and their accompanying sebaceous gland). Acne can present as non-inflammatory lesions, inflammatory lesions, or a mixture of both, affecting mostly the face but also the back and chest. The condition usually starts in adolescence, peaks at the ages of 14 to 19 years and frequently resolves by mid-twenties. The most severe forms of acne vulgaris occur more frequently in males, but the disease tends to be more persistent in females ${ }^{(3)}$. Severity of the

Received: 8/7/2018

Accepted: 17/7/2018 disease varies markedly from one individual to the other depending upon the interplay of various factors involved in the development of acne vulgaris. Grading systems based on the clinical appearance of lesions as well as "lesion counting" is useful in assessing the severity of acne vulgaris.

The influence of nutrition on skin health is a growing research area, a lot of researches done to assess the effect of various nutrition substances on the management and prevention of acne. We therefore reviewed the literature done in this filed to assess the effect of diet and nutrition substances in the management and prevention of acne vulgaris. 


\section{Pathogenesis}

Acne vulgaris occur as a result of inflammatory process inside the pilosebaceous unit. This condition mostly develops at the time of puberty when changes in the body's hormonal milieu alter pilosebaceous gland function. At the start, follicular epithelial cells differentiate abnormally and form tighter intracellular adhesions and, therefore, are shed less readily. As a result of this process hyperkeratotic plugs or microcomedone will develop, which will grow in size to form noninflammatory, closed or open comedones ${ }^{(15)}$. Furthermore, circulating androgen in the body induce sebum production, a further contributing to the development of comedones ${ }^{(16)}$. Conditions associated with increase androgen production such as, polycystic ovarian syndrome, and congenital adrenal hyperplasia are associated with further worseness of acne vulgaris ${ }^{(17)}$.

Acne vulgaris lesions distributions depend on pilosebaceous gland density and morphology and, thus, are common in regions where these structures are largest and most abundant: the face, chest, neck, and back. Non-inflammatory acne is characterized by the formation of open or closed comedones. Open comedone (black head), demonstrate dark color plugs, as a result of melanin oxidations. Closed comedones, or whiteheads, are white to flesh toned in color and seem not to have a central open pore ${ }^{(15)}$.

As a result of increase sebum production which is related to circulating androgen, a change in the skin natural flora will occur. Propionibacterium acnes, a normal component of the cutaneous flora, inhabits the pilosebaceous unit using lipid-rich sebum as a nutrient source .P acnes, therefore, flourishes in the presence of increased sebum production, leading to inflammation via complement activation and the release of metabolic byproducts, proteases, and neutrophil-attracting chemotactic factors ${ }^{(15,18)}$. When comedones are ruptured, the inflammatory acne vulgaris lesions such as papules, pustules, nodules or cyst will occur. In case of more severe cases cysts adjacent to each other coalesce and form a drainage sinus ${ }^{(15)}$.

\section{METHODOLOGY}

\section{Sample}

PubMed database was used for articles selection, and the following keys used in the mesh (("Acne Vulgaris/diet therapy"[Mesh]) AND ("Acne Vulgaris/prevention and control"[Mesh])) AND ("Acne Vulgaris/therapy"[Mesh]). A total of 143 articles were found, with further restriction by PubMed filters, and reviewing the articles titles and abstracts the final results were 8 articles. Inclusion criteria, the articles were selected based on the relevance to the project which should include one of the following topics, \{Acne vulgaris, Diet effect on acne, Acne management and control\}. Exclusion criteria, all other articles which didn't have one of these topics as their primary end point, or repeated studies.

\section{Analysis}

No software will be used, the data will be extracted based on specific form that contain (Title of the study, name of the author, objective, summary, results, and outcomes), these data will be reviewed by the group members to assess the effect of diet therapy in management and prevention of acne vulgaris. Double revision of each member's outcomes will be applied to ensure the validity and minimize the mistakes.

\section{RESULTS}

We enrolled a total of 10 studies according to our inclusion, and exclusion criteria described above. 4 of them were RCT studies, 1 cross sectional study, one case control study, and 4 reviews. All of the included study discussed about the role of nutritional substances in the management of acne vulgaris. The studies characteristics can be seen in Table 1 . 


\begin{tabular}{|c|c|c|c|}
\hline $\begin{array}{l}\text { Author/articl } \\
\text { e (Year) }\end{array}$ & Type of study & $\begin{array}{c}\text { Type of } \\
\text { Dietary } \\
\text { Interventio } \\
\mathbf{n}\end{array}$ & $\begin{array}{c}\text { Ref } \\
\text {. }\end{array}$ \\
\hline $\begin{array}{l}\text { Law MP et al. } \\
\quad(2010)\end{array}$ & Cross-sectional & Fruit juice & [4] \\
\hline $\begin{array}{l}\text { Skroza Net } \\
\text { al. (2012) }\end{array}$ & Case-control & $\begin{array}{l}\text { Fish, fruit, } \\
\text { vegetables, } \\
\text { and olive oil }\end{array}$ & [5] \\
\hline $\begin{array}{l}\text { Jung et al } \\
\text { (2014) }\end{array}$ & RCT (Double Blinded) & $\begin{array}{l}\text { Omega-3 } \\
\text { Fatty Acid } \\
\text { and } \\
\text { Gamma- } \\
\text { linolenic } \\
\text { Acid }\end{array}$ & [6] \\
\hline $\begin{array}{l}\text { P.H. Lu et al. } \\
\text { (2016) }\end{array}$ & RCT, blinded & Green tea & [7] \\
\hline $\begin{array}{c}\text { Caperton et al } \\
\text { (2014) }\end{array}$ & $\begin{array}{l}\text { RCT, Double blinded, } \\
\text { placebo controlled. }\end{array}$ & $\begin{array}{l}\text { Chocolate } \\
100 \% \text { cacao }\end{array}$ & [8] \\
\hline $\begin{array}{l}\text { Miglani A. et } \\
\text { al. (2014) }\end{array}$ & $\begin{array}{c}\text { Observational/intervention } \\
\text { al }\end{array}$ & $\begin{array}{l}\text { Arctium } \\
\text { lappa }\end{array}$ & [9] \\
\hline
\end{tabular}

\section{Fruits and Vegetables}

Law et al. ${ }^{(4)}$ investigated an association of diet and acne in a cross-sectional study in Hong Kong. Including $(n=82)$ clinical acne group and $(n=240)$ as the reference group. Intake of fresh fruit was significantly associated $(p=0.02)$ with a lower incidence of acne. ${ }^{[4]}$ Skroza N et al. (2012) carried out a case-control in Italy with acne patients $(n=93)$ and healthy individuals ( $n$ $=200$ ). Analysis of logistic regression showed that the Mediterranean diet (which contains a surplus amounts of vegetables and fruits with addition of fish and nuts) was a protective factor in this study ${ }^{(5)}$.

\section{Fish Oil}

Jung $\boldsymbol{e t}$ al. ${ }^{(6)}$ performed a randomized doubleblinded trial on omega-3 (fish oil) and Gammalinolenic Acid (GLA) on acne vulgaris patients. Forty-five Koreans, of both genders, took omega-3 and gamma-linolenic acid for ten consecutive weeks. There was significant reduction $(\mathrm{P}<0.05)$ in both acne lesions forms inflammatory and non-inflammatory- for both groups. Patient subjective assessment for acne severity was measured via VAS scores and they were significantly reduced in both groups $(\mathrm{P}<0.05)$. The histological assessment confirmed the inflammatory reduction in both groups and the IL-8 levels were significantly reduced $(\mathrm{P}<0.05)$. ${ }^{(6)}$

\section{Tea}

$\mathrm{Lu}$ and $\mathrm{Hsu}{ }^{(7)}$ observed green tea extract in the alleviation of acne in post-adolescent women ( $n$ =64). This randomized double-blinded clinical trial lasted four weeks in Taiwan. Participant females took decaffeinated green tea extract (GTE), this resulted in reduction of inflammatory acne lesions of the face ( $p=$ $0.003)$, but not in decreasing the total number of acneiform lesions $(p=0.46)$. Another interesting finding was the significant reduction in total cholesterol levels in the GTE group $(p=0.003)$ compared to the placebo one $(p=0.95)$. Nevertheless, other studies that have been done on both genders weren't double-blinded or randomized, had no control group, and included small sample sizes. However, they demonstrated a positive results of green tea extract on acne. ${ }^{(7)}$

\section{Hyperglycaemic carbohydrates (e.g. sugar,} soda, pizza, wheat, etc.)

Caperton et al. ${ }^{[8]}$ tested the effects of chocolate on patients with acneiform lesions who also had a history of acne vulgaris $(n=13)$. This randomized trial, done in the United States, was double blinded and lasted for seven days. All thirteen adult male participants received unsweetened $100 \%$ cacao. The results were statistically significant on Day $4(p=0.006)$ and 
Day $7(p=0.043)$ as total numbers of acneiform lesions -both inflammatory and noninflammatory- increased ${ }^{[8]}$

\section{Arctium Lappa}

Miglani et al. ${ }^{(9)}$ observed arctium lappa in the treatment of acne vulgaris. The uncontrolled interventional study included Indians of both genders $(n=32)$, aged twelve to twenty five, supplemented with arctium lappa followed by seven days of placebo while improvement occurred for a duration of six months. When the symptoms reemerged, an increased dose of lappa was given followed by placebo for seven days. There were statistically significant results ( $p=$ $<0.001)$ as reduction occurred in all lesions, with significant $(p=<0.001)$ improvement in acne quality of life as symptoms alleviated and this was assessed subjectively by Acne-QoL. Subjective assessment of acne severity was also significantly ( $p=<0.001)$ decreased and this was assessed by GAGS score ${ }^{(9)}$.

\begin{tabular}{|c|c|}
\hline $\begin{array}{c}\text { Author/Article } \\
\text { (Year) }\end{array}$ & Type of Dietary Intervention \\
\hline Saric et al. ${ }^{(10)}$ & Green tea and others \\
\hline Hammer ${ }^{(12)}$ & Tea tree oil \\
\hline Mahmoud et al. ${ }^{(11)}$ & $\begin{array}{l}\text { Low Glycemic Index food and } \\
\text { Refined Carbohydrates }\end{array}$ \\
\hline$\underset{(13)}{\text { Kucharska } \text { et al. }}$ & $\begin{array}{c}\text { Dairy products, omega-3 fatty acids, } \\
\text { dietary fiber, antioxidants, vitamin } \\
\text { A, zinc and iodine }\end{array}$ \\
\hline
\end{tabular}

\section{DISCUSSION}

We have searched for recent studies within the last decade with the introduction of dietary supplements on acne afflicted patients, these diets mainly were fruits, vegetables, oils, chocolate, and acritum lappa. Included as well were recent reviews on the topic. Our aim was to observe recent studies and possible dietary solutions for a rather common medical problem, acne vulgaris that affects psychological wellbeing as well. The etiological factors of acne are multifactorial with inevitable inflammation, beginning at puberty with disruption of otherwise normal hormonal secretions with genetic predisposition ${ }^{(12)}$. Propionobacterium acnes has been shown to proliferate within the pilosebaceous unit causing disruption of the skin barrier ${ }^{(13)}$. A recent case control study showed strong association between first-degree family history and development of moderate to severe acne ${ }^{(14)}$.

Varied fresh fruits and vegetables in diets were found to be protective against acne. The Mediterranean diet, consisting mainly of fresh fruits and vegetables with cheese and fish, in particular is of importance and should be recommended in acne patients. Moreover, as opposite to the previous diet, the western diet and especially fast food have been studied and they showed that these have an aggravating effect on acne. This was attributed mainly to industrially produced trans fatty acids and saturated fats that compose the main bulk of these dietary options ${ }^{(4,5,14)}$.

Recent study on fish oil, in Koreans, concluded with significant reduction in acne lesions, making these diet options suitable adjunctive terapies to acne. Jung et al. (6) investigated omega-3 fatty acids, being one of the leading studies with a possible adjuvant therapy solution for acne vulgaris ${ }^{(6)}$. The longer the duration of usage the better when it concerns acne alleviation with fish oil ${ }^{(7)}$.

One study concluded that green tea is a potential diet for alleviating inflammatory acne lesions in females. Limited number of human studies is available on green tea on acne alleviation, and despite showing the same promising results, the 
studies done on males for example didn't have as good design as the study we included. Further research with better protocols is still required to establish a full understanding of the benefits ${ }^{(8)}$.

Upon reviewing other reviews about the same topic, we found that this topic was one of the most debatable topics in dermatology. The reviews studied a variety of different dietary products to reveal their effect on acne, but they were not recently done, and included less significant study methods. Saric et al. ${ }^{(15)}$ did a recent review on green tea and other forms of tea polyphenols with their effects on acne vulgaris and sebum production. In the review they included eight studies, six were specifically on green tea. This review recommended further large sized studies on green tea as diet prevention of acne vulgaris ${ }^{(15)}$. Only one study by Jung et al. ${ }^{(16)}$, also included in the previous review, showed no significant decrease $(61 \%)$ in closure of comedones at the eight weeks after tea implementation $(\mathrm{p}<0.05)$ compared to at the initiation of the trial ${ }^{(16)}$.

Chocolate has shown a significant association factor in the exacerbation of acne symptoms ${ }^{(9)}$. This has been attributed to modulation of proinflammatory production such as cytokines, interleukin-1 $\beta$, and TNF $\alpha$. However, in a recent case control study they found no statistically significant relationship with acne vulgaris occurrence ${ }^{(10)}$. This contradictory finding may be associated to the various concentration and components of chocolate assessed in each study, or that high glycemic index food may produce a similar result. Future extensive studies with a larger study group, better protocol (especially regarding the chocolate form and component) is vital to assure a clearer -and evidence basedview to clinicians.

Acritum lappa, commonly known as burdock, which is used in traditional folk Chinese and western medicine, in the treatment of acne vulgaris was shown to improve the symptoms of acne with disappearance of lesions as well as improved quality of life. These results were attributed to the plant antioxidant and immune modulatory effects. Despite the small size and some limitations in the studies regarding this agent, this option presents itself as one of the most promising ones, however it needs better and bigger studies to fully estimate its benefits (11).

The reviews we found all agreed that low glycemic control index food had protective association against acne vulgaris. Another strong association that was reviewed was of fatty acids and omega 3 which upon intake resulted in less symptoms of acne. However, these studies included a small number of patients and concluded that further research is needed. Milk, dairy products and iodine which showed a positive association with acne was also a focus point in some reviews; this protective relationship lacks conclusive evidence and requires more studies. More extensive studies were done specially on milk and dairy products, and even the different formulas of milk were studied, this has shown that skim milk is more comedogenic than the whole milk. Some other dairy products that has shown an association with acne vulgaris were cottage cheese and ice cream. There are many inconclusive associations that had been included in other reviews, such as negative effect of chocolate (due to differences in the studies protocols regarding the chocolate composition) but this association is still one of the most believed ones not just among clinicians but even in the society. Moreover, a positive effect of higher consumption of fibers association was also shown (not a fact mainly due to lack of focused studies on the topic). This shows that this topic has not been studied thoroughly, and the sample sizes included were not enough to show a clinical significance. Moreover, some substances that have shown a 
great response in study subjects were not fully implicated clinically. This is mainly due to the lack of their full effects in the course of this disease, and the main agents that were included were Zinc, Vita-min A, Vitamin E, Selenium. However, a non-pharmacological option to help in the acne vulgaris approach is still a great way to provide the best possible health care to patients and that is one of the main reasons that this topic is being widely studied and on multiple dietary substances ${ }^{(17-19)}$.

\section{CONCLUSION}

The growing interest in this medically and socially important topic offers an access to new horizons in non-pharmacological therapy. Our literature review concluded that there are a number of studies with significant dietary options that showed promising results among patients and proved that associations do exist. However, the studies in this field face some problems, like the cultural differences in relation to diets, small sample sizes of participants, and the differences in dietary components. The future in this field appears encouraging and even more studies are being published, but larger studies should still be sought to prove more than association and include this treatment option in the international protocols when dealing with acne.

\section{REFERENCES}

1. Chan JJ, and Rohr JB (2000): Acne vulgaris: yesterday, today and tomorrow. Australas J Dermatol., 41:S69-72.

2. Smithard A, Glazebrook C, Williams $\mathbf{H}$ (2001): Acne prevalence, knowledge about acne and psychological morbidity in midadolescence: a community-based study. $\mathrm{Br} \mathrm{J}$ Dermatol., 145(2): 274-9.

3. Rook A, Burns T (2004): Rook's textbook of dermatology. 7th ed. Malden, Mass. ; Oxford: Blackwell Science.
4. Law M P (2010): An investigation of the association between diet and occurrence of acne: a rational approach from a traditional Chinese medicine perspective. Clin Exp Dermatol., 35(1): 31-5.

5. Skroza N, Tolino E, Semyonov L et al. (2010): Mediterranean diet and familial dysmetabolism as factors influencing the development of acne. Scand J Public Health, 40(5):466-74.

6. Jung JY, Kwon HH, Hong JS et al. (2014): Effect of dietary supplementation with omega-3 fatty acid and gammalinolenic acid on acne vulgaris: a randomised, double-blind, controlled trial. Acta Derm Venereol., 94(5): 521-5.

7. Lu PH, Hsu CH (2016): Does supplementation with green tea extract improve acne in post-adolescent women? A randomized, double-blind, and placebocontrolled clinical trial. Complement Ther Med., 25:159-63.

8. Caperton C, Block S, Viera $M$ et al. (2014): Double-blind, Placebo-controlled Study Assessing the Effect of Chocolate Consumption in Subjects with a History of Acne Vulgaris. J Clin Aesthet Dermatol., 7(5): 19-23.

9. Miglani A, Manchanda $R$ (2014): Observational study of Arctium lappa in the treatment of acne vulgaris. Homeopathy, 103(3): 203-207.

10. Saric S, Notay M, Sivamani R (2016): Green Tea and Other Tea Polyphenols: Effects on Sebum Production and Acne Vulgaris. Antioxidants (Basel), 6(1):2

11. Mahmood SN, Bowe WP (2014): Diet and acne update: carbohydrates emerge as the main culprit. J Drugs Dermatol., 13(4): 42835 .

12. Hammer KA (2015): Treatment of acne with tea tree oil (melaleuca) products: a review of efficacy, tolerability and potential 
modes of action. Int $\mathbf{J}$ Antimicrob Agents, 45(2): 106-10.

13. Kucharska A, Szmurlo A, Sinska B (2016): Significance of diet in treated and untreated acne vulgaris. Postepy Dermatol Alergol., 33(2): 81-86.

14. Melnik BC (2015): Linking diet to acne metabolomics, inflammation, and comedogenesis: an update. Clin Cosmet Investig Dermatol., 8:371-388.

15. Brown SK, Shalita A (1998): Acne vulgaris. Lancet, 351(9119):1871-1876.
16. Chen W, Thibouot D, Zouboulis C (2002): Cutaneous androgen metabolism: basic research and clinical perspectives. J Invest Dermatol., 119(5):992-1007.

17. Imperato-McGinley J, Gautier $\mathrm{T}$, Cai $\mathrm{L}$ et al. (1993): The androgen control of sebum production. Studies of subjects with dihydrotestosterone deficiency and complete androgen insensitivity. J Clin Endocrinol Metab., 76:524-5288.

18. Tan AW, Tan HH (2005): Acne vulgaris: a review of antibiotic therapy. Expert Opin Pharmacother., 6(3):409-418 